\title{
Making collaboration work: application of a Conceptual Design Stages Protocol for pre-BIM stages
}

\author{
M. Leon ${ }^{1}$, R. Laing ${ }^{1}$, J. Malins ${ }^{2}$ \& H. Salman ${ }^{1}$ \\ ${ }^{I}$ Scott Sutherland School of Architecture and Built Environment, \\ Robert Gordon University, UK \\ ${ }^{2}$ Norwich University of the Arts, UK
}

\begin{abstract}
This paper presents the application of a Conceptual Design Stages Protocol during feasibility stages within a multidisciplinary design team, for the purpose of bridging the gap between ideas generation and their representation in later and more advanced design stages. Increased effort during the early and conceptual design is a prerequisite for effective and sustainable overall design and construction. Consequently, shift of the effort towards feasibility stages aims to tackle problems with workflow, education and organisation of design teams at the very beginning of a project. Furthermore, the application of an organised process during conceptual design could further support a multi-party agreement, hence enhancing the potentials of collaboration and coordination for the entirety of a project. This paper demonstrates the Conceptual Design Stages Protocol as an organised process encompassing different professionals, technologies and means for communication that has been tested in a conceptual stage study and the outcomes have been incorporated within BIM. The impact of this Protocol on designers' cognitive, physical, perceptual and collaborative actions is presented and the first pre-BIM Protocol is established.

Keywords: Conceptual Design Stages Protocol, pre-BIM stages, multidisciplinary collaboration, multi-party agreement.
\end{abstract}

\section{Introduction}

This paper presents a study that examines and monitors a multidisciplinary team of final year students of the Architecture Engineering and Construction (AEC) 
industry from handing a design brief to undertake a feasibility work stage, up to deciding on the final conceptual solution, followed by a final discussion and feedback. The participants were asked to follow the Conceptual Design Stages Protocol, to employ a computational design tool applicable on Tangible User Interfaces (TUIs) and eventually to develop their conceptual solution and to make the transfer to BIM programs and Revit.

The following section of the paper defines the setting of the study, its components and structure, physical and digital design tools, and also provides a short description of the Conceptual Design Stages Protocol. The third section describes the different levels of analysis, from the activities mapping to analytical actions' coding. Finally, a short summary describes the key points of the particular paper, together with the impact and contribution of the specific research.

\section{Conceptual Design Stages Protocol and the study's components}

\subsection{Conceptual Design Stages Protocol}

Increased effort during the early and conceptual design is a prerequisite for effective and sustainable overall design and construction especially due to the 2016 UK BIM mandate. BIM is changing the way we collaborate, thus shifting the focus from the chain of activities to efficient collaboration and innovative ways of co-creating, sharing and collecting relevant information among different but project related disciplines $[3,4]$. Therefore, shift of the effort towards the early and conceptual design stages has the potential to lead to fewer problems during the later and more complex design stages, while effective collaboration among the different professionals and disciplines straight from the beginning of a project.

Leon [1] developed and tested this innovative Conceptual Design Stages Protocol, a holistic approach to conceptual design integrating different opinions and professionals, computer media and tactile technologies, organised communication and spaces for ideation. This Protocol aims to be adopted by AEC professionals during pre-BIM stages in order to address the lack of an organised system for supporting the early conceptual design stages, thus enhancing multidisciplinary collaboration and providing informed design solutions. The steps of this process are presented in Figure 1; the Conceptual Design Stages Protocol initiates with the team formation and the introduction of the brief, followed by discussion of project requirements, solution synthesis and brainstorming, solution evaluation, consensus and the final solution. Decision points that consist of small milestones are also parts of this process; these milestones reflect the shared views and agreements among the participants regarding the project, for the purpose of moving forward the design. Feedback loops allow the reconsideration of the achieved consensus in case this informed compromise does not comply with the design brief requirements, the project objectives and goals.

Furthermore, the development of this Conceptual Design Stages Protocol is based on observed problems in the AEC design processes, in relation to workflow 


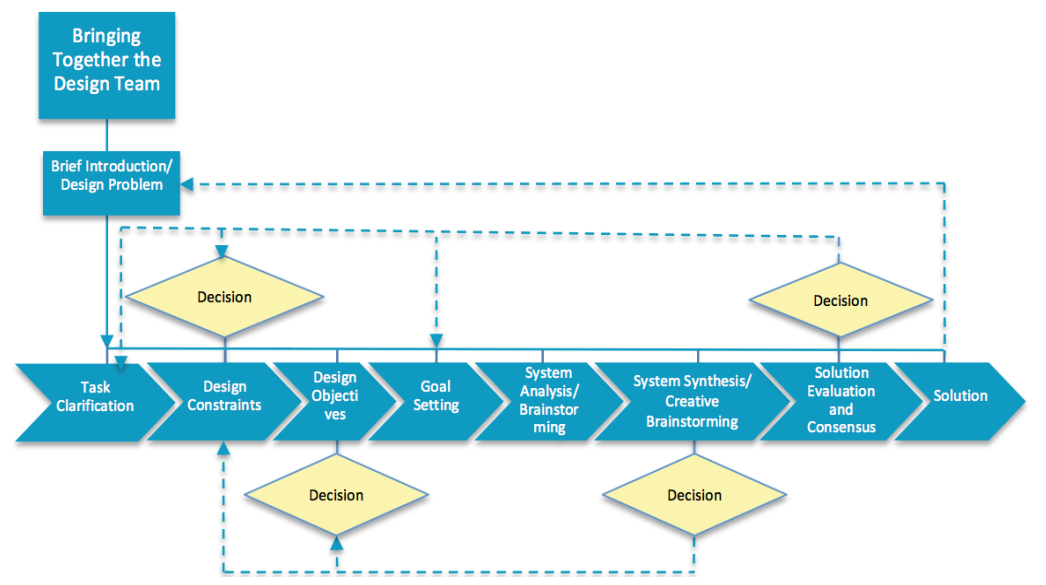

Figure 1: The developed Conceptual Design Stages Protocol.

and project governance [5-7], education, design features and conventions [8-10] and technology and collaborative software implementation [21, 22]. The Conceptual Design Stages Protocol further includes aspects like team building and design and communication management [11-13]. The development of the Protocol is influenced from relevant design processes already modelled according to different perspectives, theories and industries, from engineering $[6-8,14]$ and design $[5,15,16]$. It also clearly extends design processes within the AEC industry [17-20]. These design processes have supported the synthesis, development and testing of a pre-defined and multidisciplinary Conceptual Design Stages Protocol [1], which supports the multi-party agreement and multidisciplinary early involvement for maximising the potentials of collaboration and coordination for the entirety of a project.

The adoption of this Protocol also extended previous research on collaborative design [23-25]. The Conceptual Design Stages Protocol was evaluated within a set of three experimental studies involving multidisciplinary teams of professionals and last year students with experience in the AEC industry [1]. All three studies were video recorded, they were simulating the process a team follows after handing a design brief until the initial concepts are developed and they shared the same structure.

\subsection{Study's components}

This paper is presenting the third research study according to [1] and based on the aforementioned description. This study supported an evaluation of the Conceptual Design Stages Protocol and the developed computational design tool.

\subsubsection{Participants}

The recruited participants for the specific study were comprised of last year's students of Scott Sutherland School of Architecture and Built Environment. The team was comprised of five students, two architects, a quantity surveyor (QS), a 
building surveyor (BS) and an architectural technologist (AT). They partially knew each other from beforehand and they were all about to graduate while all of them had already some professional experience in practices. The purpose for recruiting students for this study was also to monitor the professional silos and how communication flows would be affected by multidisciplinary collaboration.

\subsubsection{Study structure and brainstorming tools}

The study was divided into three parts and two further stages within the second part, as illustrated in Figure 2, while it was being moderated by the researcher [1]. The study initiated with an introduction to the design task and a short icebreaker for the participants to familiarize themselves, followed by handing the design brief and explaining the conceptual design task. The moderator also introduced the Conceptual Design Stages Protocol at that stage and provided instructions regarding its application for the whole study duration. The design brief that was provided was about a small educational building to facilitate for the students' capacities, thus allowing them to complete the task within the given time limitations. The brief was not lacking information though. The design brief was formed into a project execution plan and it included the involved parties, budget restrictions and scope of the project with the deliverables, the project description and the space requirements. Furthermore, site and area information was also provided together with number of expected occupants and information on some basic regulations to comply with.

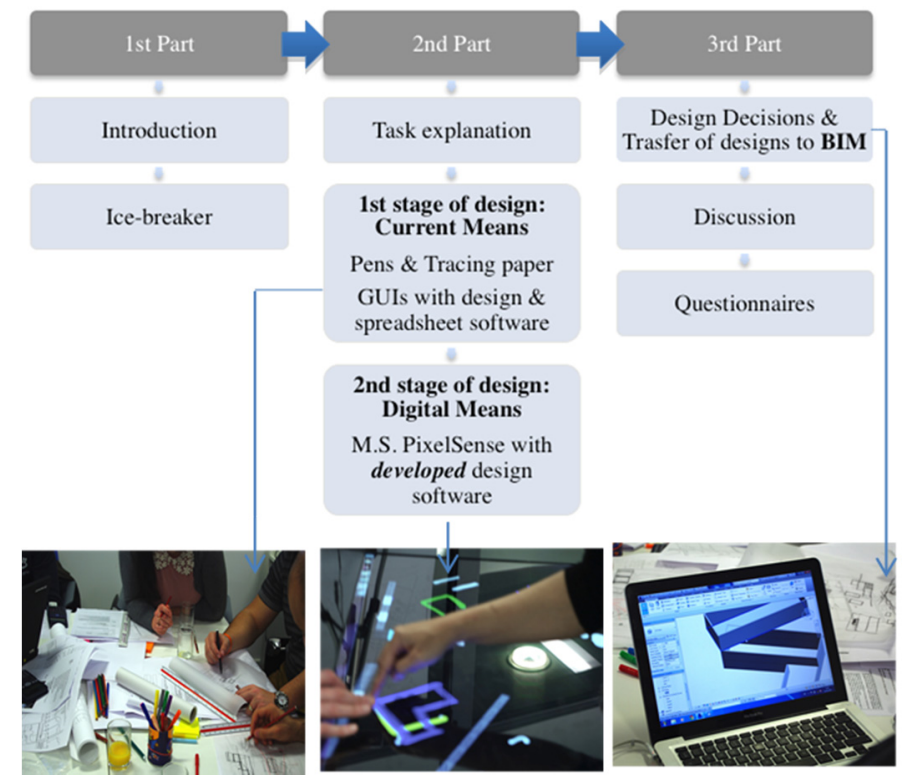

Figure 2: Study's structure: parts and stages.

Relevant educational building examples were also presented during the design brief introduction and the participants initiated immediately the design process and 
inspected the design brief, the maps and data provided in the execution plan/brief. The parts and stages had certain time slots/duration and the study moderator was informing the group on their available time to complete the task within three and a half hours. Detailed description of the study structure is provided in Figure 2.

The participants were provided with a range of physical and digital tools. The digital means and physical design mediums were utilised at different stages of the study. The conceptual design took place during the second part of the study, by utilising physical means during the first stage (the current paradigm of conceptual design), and afterwards by making use of digital means and a specifically developed design software during the second stage. The transfer of these decisions into BIM occurred during the third part of the study, as illustrated in Figure 2.

The physical design mediums included drafting and drawing tools, a flip-chart painting surface, tracing paper, pencils, markers and maps of the area specified in the design brief. Moreover, brainstorming tools were also available, like post-its and a magnetic board with hexagonal pieces for mind mapping purposes. A laptop was provided with Internet access and relevant software was installed, i.e. Microsoft Excel for calculations, Revit and AutoCAD.

A TUI was used for the second stage of the study, which was a MS PixelSense with a specifically developed conceptual design application. This conceptual design application applicable to a computer mediated environment (TUI) was employed to be a facilitator for collaborative design, thus helping multidisciplinary professionals work together efficiently and effectively by supporting ideation processes. This augmented reality environment aimed to further support collaborative design and extend relevant research in the field, from the Electronic Cocktail Napkin [30], to more recent multi-sensory input [31, 32]. Further details on the specific application can be found in $[1,2]$.

\section{Study's results}

The analysis of the study involved examining audio and video recordings of the whole duration of it. Two different methods were applied for the analysis of the audio and video; the activities mapping and the protocol analysis. The first method allows mapping the evolution of the design process of the studies within time [26]. The second one is a macroscopic analysis for identifying participants problem solving and cognitive actions, identifying collaboration actions and monitor participants interactions with computer media and physical design mediums, a methodological approach first established from Gero and McNeill [27] and further adapted from Gabriel and Maher [28] and Gu et al. [29]. As a result, observations on the effectiveness of the intended use of the Conceptual Design Stages Protocol and the impact of the computational design tool on conceptual design could be mapped and monitored.

\subsection{Protocol analysis: activities mapping}

The first level of analysis focuses on mapping the activities that the team followed within the duration of the study. Based on that method, an activities map was 
created, as presented in Figure 3. The chart followed the step of the given predefined Conceptual Design Stages Protocol and it monitored how closely the team of participants followed the Protocol or whether they adapted it to their requirements.

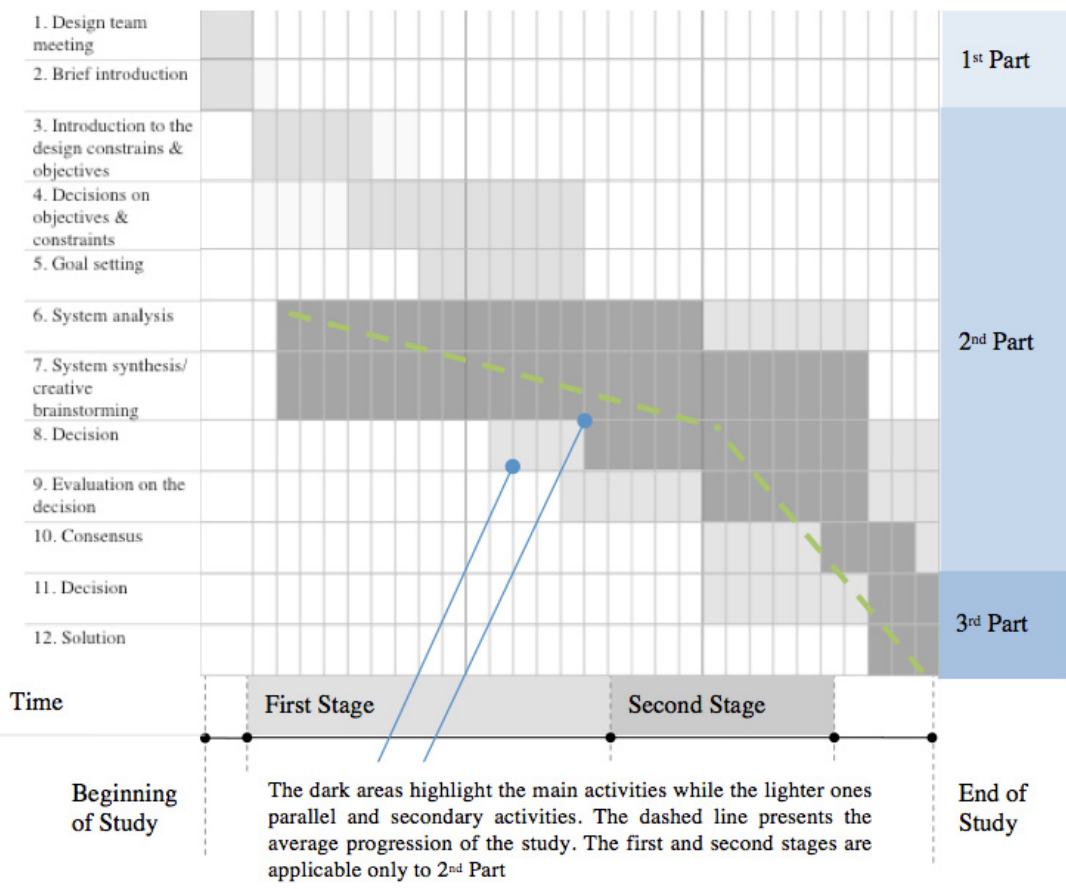

Figure 3: The design activities progression during the study.

Shortly after the introductory first part of the study, the second part initiated with the participants having already familiarised themselves with the available design mediums, they were examining the details of the design brief and taking notes of information they considered important. During the beginning of the first stage of the second part, communication was limited to individual inspections of the design brief. Soon after, the architects and AT started designing while the QS and BS were exploring aspects relevant to cost. The discussions were following a system synthesis and brainstorming process straight for the beginning, with discussions about forms, shapes and spaces connections and locations being discussed from the start, accompanied by sketches and notes. The team commenced the study with a holistic approach to their conceptual design process by considering multiple steps at the same time, including discussing on possible solutions, sketching and synthesising their ideas and afterwards comparing them to the objectives as set by the design brief. They did not question the design brief and, additionally, they did not add further information to it or try to clarify aspects. Communication among the participants was intense straight from the beginning as well, with all the different disciplines participating and questions being asked 
among them for clarifications on topics like the budget, the building's potential shapes and building regulations.

The moderator prompted the participants to utilise the MS PixelSense for their design explorations during the second stage of the second part of the study. The team had already found a basic form, an initial budget and other design details, features relevant to the circulation space, interior space and cost limitations. An introduction to the TUI assisted the team to make a smoother transition to the design environment and they initiated using the design application with a great ease. The team kept analysing the conceptual ideas during that stage, with a greater multidisciplinary communication this time, since the table top environment allowed for an easier shared understanding of the designs. Both 2D and 3D visualisations of the ideas together with intense dialogues among them assisted in communicating the concepts of the design and promoted questions and further clarifications of the developed ideas, together with greater elaboration on nonclarified topics, regarding the levels, people's flow and constructability. Perceptual activities were enhanced due to shared understanding of the ideas through the MS PixelSense and collaboration was promoted. During that stage, many different issues with their concepts were resolved, design decisions were taken and by the end of that stage they were ready to make a leap in design and transfer their concepts in BIM software.

Eventually, the third part of the study was focused on finalising the conceptual design, transferring the information into BIM and reflecting back on the whole duration of the study. During this part, intense negotiations took place among the different disciplines for finalising design, constructability and cost, while design problems occurred due to the greater detail of design. These problems were acknowledged as part of the detailed design and soon after the study came to a halt since the conceptual design was completed.

The team was comprised out of last year students, and the limitations on their experience was evident from the beginning, with the process initiating straight from the system synthesis and analysis instead of clarifying their objectives and constraints from the beginning. They managed to cover up though since during the brainstorming process they were making often iterations between brainstorming, reflection of the design brief and possible restrictions. The open communication among them also made up for the lack of experience; the design was partially led by the architects but with open and free communication and collaboration among the different disciplines and a clear appreciation and acknowledgement of the multidisciplinary input.

The process was linear but it did not initiate from deciding on objectives and constraints as such, since the participants did not elaborate on the design brief in the beginning, rather they instantly started brainstorming on potential design solutions. The design objectives and constraints as specified from the project execution plan were guiding their decisions during the first half of the first stage. Soon after though, the team members were adapting that information according to their professional viewpoints and were adjusting the design objectives to their project. A reason for that is the lack of experience among the design team members. Multiple steps were being undertaken though simultaneously, including 
brainstorming and evaluation of their ideas while moving between deciding on design aspects and synthesising information. This process lasted for whole second part of the study and the final design consensus among the team members was achieved during the middle of the third part of the study.

\subsection{Protocol analysis: actions' coding}

The second level of analysis is providing feedback on the participants' interactions among them and with the physical and digital media together with their cognitive, perceptual, conceptual and physical actions during each stage of the study according to the structure presented in Figure 2.

A comparison of the activities' duration in the two stages of the second part of the study showcased the differences between the use of physical design mediums and digital media. The conceptual design and collaborative activities were quite intense overall. The comparison between the two stages is presented in Figure 4.

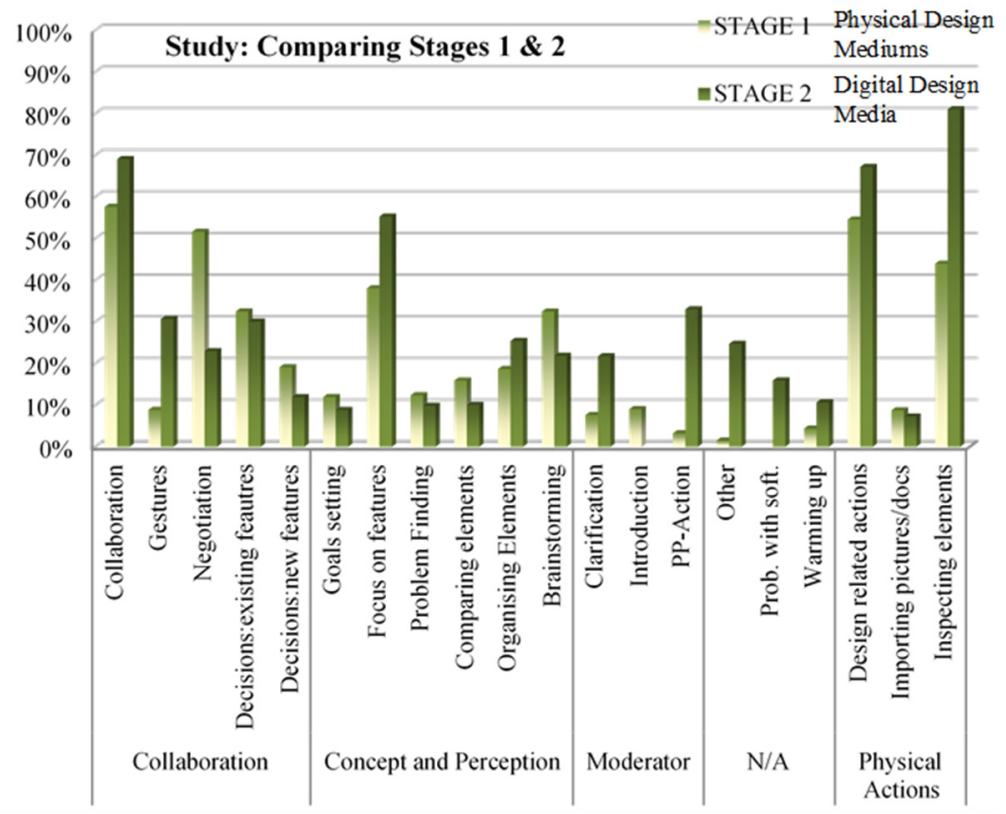

Figure 4: Comparing actions' duration for first and second stage of the study.

The participants followed the process of Conceptual Design Stages Protocol overall. The team moved quickly to the brainstorming steps and the dynamics of the participants led them to separate to two smaller teams, the one of the designers and the one of the surveyors. This separation lasted for parts of the brainstorming session and the reason was for them to tackle faster the project requirements and face simultaneously design, cost and constructability issues. The process did work and the two smaller teams were coming together quite often to share opinions and 
understanding and to decide on different aspects of the project. Collaboration, negotiations and decisions on various new and developing ideas together with intense design and inspection were the most prominent aspects of that stage, with durations that were lasting for $58 \%$ of the time for collaboration, $52 \%$ for negotiations, $32 \%$ for decisions making, 53\% for design and $43 \%$ for inspection of design elements. Moderator's activities were quite low and subtle during that stage (lasting for $2 \%$ up to $8 \%$ of the time) and the process was moving forward rapidly and smoothly.

Overall during the first stage, the participants decided on various aspects of the project but the finalisation of their ideas occurred during the second stage, while using the MS PixelSense. The majority of the actions had an increased duration within the second stage, with the peak of percentages including collaboration, lasting for $69 \%$ of the stage duration, design and elements inspection with $64 \%$ and $80 \%$ accordingly, greater negotiations (52\%) and brainstorming (53\%). The participants focused around the tangible design surface that led to more active collaboration and vigorous ideas exchange while designing new and developed concepts. The actual design process on top of the TUI promoted their ideation process and the simultaneous multidisciplinary discussions. However, during that stage a number of technical problems occurred, which led to a greater interference of the moderator, with $21 \%$ of the time required for clarifications and $31 \%$ for promoting their design process and asking them to participate. Furthermore, the multidisciplinary dialogues even though intense, they were stalling at points, again an aspect that required the moderator to support the group for keeping it in track with the Conceptual Design Stages Protocol.

The study concluded with questionnaires intended to provide feedback for the overall study process and the participants' experience. Participants were pleased with the study and the percentages of their satisfaction (with a maximum of $100 \%$ ) were quite high for all the different aspects that were asked. Their unanimous greatest positive feedback came from the acknowledgement that the group benefited from multidisciplinary working $(100 \%)$, followed by the effectiveness of the group decisions (96\%) and the efficient contribution from all the team members $(96 \%)$. The lowest feedback was on group organisation $(76 \%)$; the team was happy with the teamwork but realised that they could have been even more effective during the study. Furthermore, they were happy with the end solution they produced and they believed it answered the design brief.

The second part of the questionnaire was focused on the use of the Conceptual Stages Design Protocol. The average feedback was very strong and the participants found the overall collaborative Protocol efficient, useful and helpful. The greatest levels of satisfaction (100\% and 96\%) were observed regarding the details included in the design brief for the required task, the use of the evaluation tool for assessing the produced conceptual design, the steps that were guiding them during the process and the fact that the Protocol was a realistic description of reality for conceptual design. Very positive feedback was monitored in relation to the usefulness of various aspects like the brainstorming tools, the design Protocol in its current form and the assistance it provided for collaboration. The smaller percentages of satisfaction $(76 \%)$ were related to the use of examples in the 
beginning of the process and the project specifications, the reason being that they were already looking for relevant examples themselves and that they did not consider that they had enough time to further adapt their project to strict sustainability specifications.

\section{Conclusions and discussion}

The analysis showed that the application of the Protocol for pre-BIM phases led to enhanced collaboration among the participants and improved cognitive and conceptual activities. The process allowed for the promotion of ideation together with advanced solutions in a reduced amount of time, and an increased satisfaction of the participants demonstrating that the Protocol makes a significant improvement of the design process and leads to more effective teamwork and communication.

The presented study evaluated the Conceptual Design Stages Protocol and the developed computational design software. The whole duration of the study was closely monitored and the impact of the Conceptual Design Stages Protocol and TUIs was examined. Furthermore, the participants provided feedback regarding the process and the design application and they were comprised out of last year students with a limited professional experience.

Regarding the application of the Protocol, it was apparent that they followed it quite close and they reached a final result through intense collaborative, conceptual, perceptual and physical activities. Communication was strong throughout the study and the discussions were flowing among the different disciplines, with a limited number of clashes and a more cooperative approach. Design was informed from the multidisciplinary feedback and the participants were reaching out to their colleagues for sharing opinions, information and ideas and getting feedback. As a result, the designs evolved constructively up to the beginning of detailed design within Revit.

The application of MS PixelSense for design purposes further supported the design process, thus providing the suitable environment for an uninterrupted engagement with the evolution of the conceptual stage. The participants were enthused with the capabilities of the TUI and they found potentials for its application within the construction industry for supporting a smoother and instant collaboration. Additionally, the comments were supported from the protocol analysis results that demonstrated the enhancement of design, collaborative and cognitive activities compared to the first stage when using physical design mediums. The TUI eventually promoted a smooth design and cognition continuum, thus encouraging the finalisation of their conceptual design.

The fact that the participants were students led to a more active approach to design and collaboration; the participants had no barriers during the collaboration that was open and unrestricted. Furthermore, they started designing from the very beginning of the study, which allowed ideas' exchange for a great number of potential design solutions within the multidisciplinary context.

Overall, the Conceptual Design Stages Protocol is highly adaptable and it represents a collaborative design process that could be applied at any point within 
the different stages of design. Additionally, the type of participants could further adapt according to the type of procurement utilised for a project. The duration of this process and its milestones are not restricted but it could be modified according to the requirements of a project. Regarding the application of the process, it could be facilitated by design, collaboration or project managers and it could easily be integrated within an ongoing or a new project.

\section{References}

[1] M. Leon, "Development of a Computer Mediated Multidisciplinary Design Protocol and its Application to the Early Architectural Design Stages," PhD Thesis, RGU, Aberdeen. 2015.

[2] M. Leon, C.D. Doolan, R. Laing, J. Malins and H. Salman, “Application of Interactive Surfaces to Support Computer Mediated Collaborative Design Environment," in Proceedings of the 18th International Conference on Information Visualisation, July, 2014.

[3] M. Kassem, N. Iqbal, G. Kelly, S. Lockley and N. Dawood, "Building information modelling: protocols for collaborative design processes," Journal of Information Technology in Construction (ITcon), vol. 19, pp. 126-149, 2014.

[4] PAS1192-2:2013, Specification for information management for the capital/delivery phase of construction projects using building information modelling. Technical report, 2013.

[5] Cross, N., Engineering Design Methods - Strategies for Product Design. John Wiley \& Sons: West Sussex, England, 4th edition, 2008.

[6] Chestnut, H., Systems Engineering Tools. John Wiley \& Sons Inc.: New York, 1965.

[7] Pahl, G. \& Beitz, W., Engineering design: a systematic approach. Springer: Berlin, Germany, 2nd edition, 1995.

[8] Daenzer, W.F., Systems Engineering. Industrielle Organisation Zürich: Zurich, revision edition, 2002.

[9] Snow, C.P., The Two Cultures. Cambridge University Press: Cambridge, 1993.

[10] Rittel, H., Second-generation design methods. Developments in Design Methodology, pp. 317-327, 1984.

[11] Anderson, N.R. \& West, M.A., Measuring climate for work group innovation: development and validation of the team climate inventory. Journal of Organizational Behavior, 19(3), pp. 235-258, 1998.

[12] Fischer, G., Symmetry of ignorance, social creativity, and meta-design. Knowledge-Based Systems, 13(7-8), pp. 527-537, 2000.

[13] Dainty, A., Moore, D. \& Murray, M., Communication in Construction. Taylor \& Francis: Oxon, 2006.

[14] Krick, V., An introduction to Engineering and Engineering Design. Wiley \& Sons Inc.: New York, London, Sydney, Toronto, 2nd edition, 1969.

[15] Schon, D., The Reflective Practitioner: How Professionals Think in Action. Ashgate Publishing Limited: London, 1991. 
[16] Valkenburg, R. \& Dorst, K., The reflective practice of design teams. Design Studies, 19(3), pp. 249-271, 1998.

[17] Rod, M., Subjective personal introspection in action oriented research. Qual. Research in Orgs \& Mgmt, 6(1), pp. 6-25, 2011.

[18] East, E.W., Construction operations building information exchange (COBie), 2013.

[19] BS 7000-4:2013, Design management systems. Guide to managing design in construction. Technical report, British Standards Institution, 2013.

[20] Sinclair, D., (ed.) RIBA Plan of Work 2013. RIBA: London, 2013.

[21] P. Dourish and G. Bell, "The infrastructure of experience and the experience of infrastructure: meaning and structure in everyday encounters with space," Environment and Planning B: Planning and Design, vol. 34, pp. 414-430, 2007.

[22] W. Shen, Q. Hao and W. Li, “Computer supported collaborative design: Retrospective and perspective," Comput. Ind., vol. 59, pp. 855-862, 12. 2008.

[23] S. Ben Rajeb, C. Lecourtois and F. Guena, "Operations of Conception in Architectural Collaborative Design," in Future Cities: 28th eCAADe Conference Proceedings, pp. 687-695, 2010.

[24] P. Badke-Schaub and E. Frankenberger, "Analysing and modelling cooperative design by the critical situation method," Travail Humain, vol. 65, p. 293, 2002.

[25] T. Dorta, E. Pérez and A. Lesage, "The ideation gap: hybrid tools, design flow and practice," Des Stud, vol. 29, pp. 121-141, 3. 2008.

[26] S. Austin, J. Steele, S. Macmillan, P. Kirby and R. Spence, "Mapping the conceptual design activity of interdisciplinary teams," Des Stud, vol. 22, pp. 211-232, 5. 2001.

[27] J.S. Gero and T. McNeill, "An approach to the analysis of design protocols," Des Stud, vol. 19, pp. 21-61, 1. 1998.

[28] G.C. Gabriel and M.L. Maher, "Coding and modelling communication in architectural collaborative design," Autom. Constr., vol. 11, pp. 199-211, 2. 2002.

[29] N. Gu, M.J. Kim and M.L. Maher, "Technological advancements in synchronous collaboration: The effect of 3D virtual worlds and tangible user interfaces on architectural design," Autom. Constr., vol. 20, pp. 270-278, 5. 2011.

[30] M.D. Gross and D. Yi-Luen, "Ambiguous intentions: a paper-like interface for creative design," in Proceedings of the 9th annual ACM symposium on User interface software and technology, pp. 183-192, 1996.

[31] M.J. Kim and M.L. Maher, "The impact of tangible user interfaces on spatial cognition during collaborative design," Des Stud, vol. 29, pp. 222-253, 5. 2008.

[32] S. Zhen, R. Blagojevic and B. Plimmer, "Tangeo: geometric drawing with tangibles on an interactive table-top," in CHI 2013 Extended Abstracts on Human Factors in Computing Systems (CHI EA 2013), pp. 1509-1514, 2013. 